\title{
Childhood Undifferentiated High Grade Pleomorphic Sarcoma of Bone
}

National Cancer Institute

\section{Source}

National Cancer Institute. Childhood Undifferentiated High Grade Pleomorphic Sarcoma

of Bone. NCl Thesaurus. Code C114750.

An undifferentiated high grade pleomorphic sarcoma that arises from the bone and occurs during childhood. 